\title{
Small generating sets for the Torelli group
}

\author{
ANDREW PUTMAN
}

\begin{abstract}
Proving a conjecture of Dennis Johnson, we show that the Torelli subgroup $\mathcal{I}_{g}$ of the genus $g$ mapping class group has a finite generating set whose size grows cubically with respect to $g$. Our main tool is a new space called the handle graph on which $\mathcal{I}_{g}$ acts cocompactly.
\end{abstract}

20F05; 20F38, 57M07, 57N05

\section{Introduction}

Let $\Sigma_{g, n}$ be a compact connected oriented genus $g$ surface with $n$ boundary components. The mapping class group of $\Sigma_{g, n}$, denoted $\operatorname{Mod}_{g, n}$, is the group of orientationpreserving homeomorphisms of $\Sigma_{g, n}$ that fix the boundary pointwise modulo isotopies that fix the boundary pointwise. We will often omit the $n$ if it vanishes. For $n \leq 1$, the Torelli group, denoted $\mathcal{I}_{g, n}$, is the kernel of the action of $\operatorname{Mod}_{g, n}$ on $\mathrm{H}_{1}\left(\Sigma_{g, n} ; \mathbb{Z}\right)$. The Torelli group has been the object of intensive study ever since the seminal work of Dennis Johnson in the early '80's. See [10] for a survey of Johnson's work.

Finite generation of Torelli One of Johnson's most celebrated theorems says that $\mathcal{I}_{g, n}$ is finitely generated for $g \geq 3$ and $n \leq 1$ (see [9]). This is a surprising result though $\operatorname{Mod}_{g, n}$ is finitely presentable, $\mathcal{I}_{g, n}$ is an infinite-index normal subgroup of $\operatorname{Mod}_{g, n}$, so there is no reason to hope that $\mathcal{I}_{g, n}$ has any finiteness properties. Moreover, McCullough and Miller [13] proved that $\mathcal{I}_{2, n}$ is not finitely generated for $n \leq 1$, and later Mess [14] proved that $\mathcal{I}_{2}$ is an infinite rank free group.

Johnson's generating set Johnson's generating set for $\mathcal{I}_{g, n}$ when $g \geq 3$ and $n \leq 1$ is enormous. Indeed, for $\mathcal{I}_{g}$ (resp. $\mathcal{I}_{g, 1}$ ), it contains $9 \cdot 2^{2 g-3}-4 g^{2}+2 g-6$ (resp. $\left.9 \cdot 2^{2 g-3}-4 g^{2}+4 g-5\right)$ elements. In [11], Johnson proved that the abelianization of $\mathcal{I}_{g}$ (resp. $\left.\mathcal{I}_{g, 1}\right)$ has rank $\frac{1}{3}\left(4 g^{3}+5 g+3\right)$ (resp. $\frac{1}{3}\left(4 g^{3}-g\right)$ ). These give large lower bounds on the size of generating sets for $\mathcal{I}_{g, n}$; however, there is a huge gap between this cubic lower bound and Johnson's exponentially growing generating set. At the end of [9] and in [10, page 168], Johnson conjectures that there should be a generating set for $\mathcal{I}_{g, n}$ whose size grows cubically with respect to the genus. Later, in 
[4, Problem 5.7] Farb asked whether there at least exists a generating set whose size grows polynomially.

Main theorem In this paper, we prove Johnson's conjecture. Our main theorem is as follows.

Theorem A For $g \geq 3$, the group $\mathcal{I}_{g}$ has a generating set of size at most $57\left(\begin{array}{l}g \\ 3\end{array}\right)$ and the group $\mathcal{I}_{g, 1}$ has a generating set of size at most $57\left(\begin{array}{l}g \\ 3\end{array}\right)+2 g+1$.

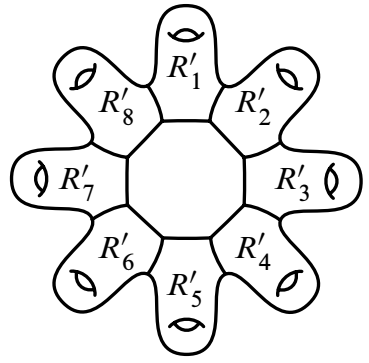

(a)

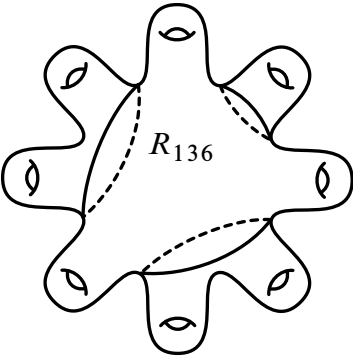

(b)

Figure 1: (a) The subsurfaces $R_{i}^{\prime} \cong \Sigma_{1,1}$ To avoid cluttering the picture, the portion of the boundaries of the $R_{i}^{\prime}$ which lie on the back side the figure are not drawn.

(b) A subsurface isotopic to $R_{136}$

The generating set we construct was conjectured to generate $\mathcal{I}_{g, n}$ by Brendle and Farb [2]. To describe it, we must introduce some notation. As in Figure 1(a), let $R_{1}^{\prime}, \ldots, R_{g}^{\prime}$ be $g$ subsurfaces of $\Sigma_{g}$ each homeomorphic to $\Sigma_{1,1}$ such that the following hold. Interpret all indices modulo $g$.

- If $1 \leq i<j \leq g$ satisfy $i \notin\{j-1, j+1\}$, then $R_{i}^{\prime} \cap R_{j}^{\prime}=\varnothing$.

- For all $1 \leq i \leq g$, the intersection $R_{i}^{\prime} \cap R_{i+1}^{\prime}$ is homeomorphic to an interval.

For $1 \leq i<j<k \leq g$, define a subsurface $R_{i j k}$ of $\Sigma_{g}$ by $R_{i j k}=\overline{\Sigma_{g} \backslash \bigcup_{l \neq i, j, k} R_{l}^{\prime}}$. Thus $R_{i j k}$ is a genus 3 surface with at most 3 boundary components such that $R_{i}^{\prime}, R_{j}^{\prime}, R_{k}^{\prime} \subset R_{i, j, k}$ (see Figure $1(\mathrm{~b})$ ).

If $S$ is a subsurface of $\Sigma_{g}$, define $\operatorname{Mod}\left(\Sigma_{g}, S\right)$ to be the subgroup of $\operatorname{Mod}_{g}$ consisting of mapping classes that can be realized by homeomorphisms supported on $S$ and $\mathcal{I}\left(\Sigma_{g}, S\right)$ to equal $\mathcal{I}_{g} \cap \operatorname{Mod}\left(\Sigma_{g}, S\right)$. The key result for the proof of Theorem A is the following theorem. 
Theorem B For $g \geq 3$, the group $\mathcal{I}_{g}$ is generated by the set

$$
\bigcup_{1 \leq i<j<k \leq g} \mathcal{I}\left(\Sigma_{g}, R_{i j k}\right) \text {. }
$$

Using Johnson's work, it is easy to see that $\mathcal{I}\left(\Sigma_{g}, R_{i j k}\right)$ is finitely generated by a generating set with at most 57 generators (see Lemma 2.2). Also, standard techniques (see Lemma 2.1) show that if $\mathcal{I}_{g}$ has a generating set with $k$ elements, then $\mathcal{I}_{g, 1}$ has a generating set with $k+2 g+1$ elements. Since there are $\left(\begin{array}{l}g \\ 3\end{array}\right)$ subsurfaces $R_{i j k}$, Theorem A follows from Theorem B.

Remark To illustrate the relative sizes of our generating sets, Johnson's generating set for $\mathcal{I}_{20}$ contains more than one trillion elements while our generating set for $\mathcal{I}_{20}$ has 64980 elements.

New proof of Johnson's theorem Our deduction of Theorem A from Theorem B depends on Johnson's theorem that $\mathcal{I}_{3}$ is finitely generated. However, Hain [6] has recently announced a direct conceptual proof that $\mathcal{I}_{3}$ is finitely generated. Hain's proof uses special properties of the moduli space of genus 3 Riemann surfaces and cannot be easily generalized to $g>3$. Combining this with our paper, we obtain a new proof that $\mathcal{I}_{g, n}$ is finitely generated for $g \geq 3$ and $n \leq 1$.

Our new proof is more conceptual than Johnson's original one. To illustrate this, we will sketch Johnson's proof. He starts by writing down an enormous finite subset $S \subset \mathcal{I}_{g, n}$ which is known (from work of Powell [15]) to normally generate $\mathcal{I}_{g, n}$ as a subgroup of $\operatorname{Mod}_{g, n}$. Letting $T$ be a standard generating set for $\operatorname{Mod}_{g, n}$, Johnson then proves via a laborious computation that for $t \in T$ and $s \in S$, the element $t s t^{-1} \in \mathcal{I}_{g, n}$ can be written as a word in $S$. This implies that the subgroup $\Gamma$ of $\mathcal{I}_{g, n}$ generated by $S$ is a normal subgroup of $\operatorname{Mod}_{g, n}$, and thus that $\Gamma=\mathcal{I}_{g, n}$.

Remark Our proof of Theorem B appeals to a theorem of [17] whose proof depends on Johnson's theorem. However, Hatcher and Margalit [12] have recently given a new proof of this result that is independent of Johnson's work.

Nature of generators Some basic elements of $\mathcal{I}_{g, n}$ are as follows (see, eg [16]). If $x$ is a simple closed curve on $\Sigma_{g, n}$, then denote by $T_{x} \in \operatorname{Mod}_{g, n}$ the Dehn twist about $x$. If $x$ is a separating simple closed curve, then $T_{x} \in \mathcal{I}_{g, n}$; these are called separating twists. If $x$ and $y$ are disjoint homologous nonseparating simple closed curves, then $T_{x} T_{y}^{-1} \in \mathcal{I}_{g, n}$; these are called bounding pair maps. Following work 
of Birman [1], Powell [15] proved that $\mathcal{I}_{g, n}$ is generated by bounding pair maps and separating twists for $g \geq 1$ and $n \leq 1$ (see [16] and [12] for alternate proofs). Johnson's finite generating set for $\mathcal{I}_{g, n}$ for $g \geq 3$ and $n \leq 1$ consists entirely of bounding pair maps. It follows easily from our proofs of Lemma 2.1 and 2.2 that our generating set consists of bounding pair maps and separating twists; see the remark after Lemma 2.2.

The handle graph Our proof of Theorem B is topological. To prove that a group $G$ is finitely generated, it is enough to find a connected simplicial complex upon which $G$ acts cocompactly with finitely generated stabilizers. We use a variant on the curve complex. If $\gamma$ is an oriented simple closed curve on $\Sigma_{g}$, then denote by $[\gamma] \in \mathrm{H}_{1}\left(\Sigma_{g} ; \mathbb{Z}\right)$ its homology class. Also, if $\gamma_{1}$ and $\gamma_{2}$ are isotopy classes of simple closed curves on $\Sigma_{g}$, then denote by $i_{g}\left(\gamma_{1}, \gamma_{2}\right)$ their geometric intersection number, ie the minimal possible number of intersections between two curves in the isotopy classes of $\gamma_{1}$ and $\gamma_{2}$. Finally, denote by $i_{a}(\cdot, \cdot)$ the algebraic intersection pairing on $\mathrm{H}_{1}\left(\Sigma_{g} ; \mathbb{Z}\right)$.

Definition Let $a, b \in \mathrm{H}_{1}\left(\Sigma_{g} ; \mathbb{Z}\right)$ satisfy $i_{a}(a, b)=1$. The handle graph associated to $a$ and $b$, denoted $\mathcal{H}_{a, b}$, is the graph whose vertices are isotopy classes of oriented simple closed curves on $\Sigma_{g}$ that are homologous to either $a$ or $b$ and where two vertices $\gamma_{1}$ and $\gamma_{2}$ are joined by an edge exactly when $i_{g}\left(\gamma_{1}, \gamma_{2}\right)=1$.

We will show that $\mathcal{H}_{a, b} / \mathcal{I}_{g}$ consists of a single edge (see Lemma 5.2) and that $\mathcal{H}_{a, b}$ is connected for $g \geq 3$ (see Lemma 3.1).

A complication It would appear that we have all the ingredients in place to use the space $\mathcal{H}_{a, b}$ to prove that $\mathcal{I}_{g}$ is finitely generated. However, there is one remaining complication. Namely, we do not know the answer to the following question.

Question 1.1 For some $g \geq 4$, let $\gamma$ be the isotopy class of a nonseparating simple closed curve on $\Sigma_{g}$. Is the stabilizer subgroup $\left(\mathcal{I}_{g}\right)_{\gamma}$ of $\gamma$ finitely generated?

In other words, we do not know if the vertex stabilizer subgroups of the action of $\mathcal{I}_{g}$ on $\mathcal{H}_{a, b}$ are finitely generated. Nonetheless, in Section 4 we will prove a weaker statement that suffices to prove Theorem B. The proof of Theorem B is in Section 5.

Smaller generating sets A positive answer to Question 1.1 would likely lead to a smaller generating set for $\mathcal{I}_{g}$, though of course this depends on the nature of the finite generating sets for the stabilizer subgroups. Let us describe one way this could work. For $g \geq 3$, let $\sigma_{g}$ be the smallest cardinality of a generating set for $\mathcal{I}_{g}$. Consider 
$g \geq 4$, and fix an edge $\{\alpha, \beta\}$ of $\mathcal{H}_{a, b}$. The proof of Theorem B shows that $\mathcal{I}_{g}$ is generated by $\left(\mathcal{I}_{g}\right)_{\alpha} \cup\left(\mathcal{I}_{g}\right)_{\beta}$. Let $S$ be a subsurface of $\Sigma_{g}$ such that $S \cong \Sigma_{g-1,1}$ and $\alpha \cup \beta \subset \Sigma_{g} \backslash S$. We have $\mathcal{I}\left(\Sigma_{g}, S\right) \cong \mathcal{I}_{g-1,1}$ (see Section 2) and $\mathcal{I}\left(\Sigma_{g}, S\right) \subset\left(\mathcal{I}_{g}\right)_{\alpha}$ and $\mathcal{I}\left(\Sigma_{g}, S\right) \subset\left(\mathcal{I}_{g}\right)_{\beta}$. Assume that there exists a finite set $V_{\alpha}$ (resp. $\left.V_{\beta}\right)$ such that $\left(\mathcal{I}_{g}\right)_{\alpha}$ (resp. $\left.\left(\mathcal{I}_{g}\right)_{\beta}\right)$ is generated by $\mathcal{I}\left(\Sigma_{g}, S\right) \cup V_{\alpha}$ (resp. $\left.\mathcal{I}\left(\Sigma_{g}, S\right) \cup V_{\beta}\right)$. The group $\mathcal{I}_{g}$ is then generated by $\mathcal{I}\left(\Sigma_{g}, S\right) \cup V_{\alpha} \cup V_{\beta}$. Lemma 2.1 says that $\mathcal{I}\left(\Sigma_{g}, S\right) \cong \mathcal{I}_{g-1,1}$ can be generated by $\sigma_{g-1}+2 g+1$ elements. Moreover, it seems likely that there exists some relatively small $K$ such that $\left|V_{\alpha}\right|,\left|V_{\beta}\right| \leq K g^{2}$. This would imply that

$$
\sigma_{g} \leq \sigma_{g-1}+2 g+1+2 K g^{2} \text {. }
$$

Iterating this, we would get that

$$
\sigma_{g} \leq \sigma_{3}+\sum_{i=4}^{g}\left(2 i+1+2 K i^{2}\right)
$$

for $g \geq 4$. This bound is cubic in $g$ (as it needs to be), but as long as $K$ is not too large it is much smaller than $57\left(\begin{array}{l}g \\ 3\end{array}\right)$.

Finite presentability Perhaps the most important open question about the combinatorial group theory of $\mathcal{I}_{g}$ is whether or not it is finitely presentable for $g \geq 3$. One way of proving that a group $G$ is finitely presentable is to construct a simply-connected simplicial complex $X$ upon which $G$ acts cocompactly with finitely presentable stabilizer subgroups (see, eg [3]). For example, Hatcher and Thurston use this technique in [7] to prove that the mapping class group is finitely presentable.

The handle graph $\mathcal{H}_{a, b}$ appears to be the first example of a useful space upon which $\mathcal{I}_{g}$ acts cocompactly (of course, there are trivial non-useful examples of such spaces; for example, the Cayley graph of $\mathcal{I}_{g}$ or a 1 -point space). Unfortunately, while $\mathcal{H}_{a, b}$ is connected for $g \geq 3$, it is not simply connected. Indeed, it does not even have any 2 -cells (and is not a tree). However, one could probably attach 2-cells to $\mathcal{H}_{a, b}$ to obtain a simply connected complex upon which $\mathcal{I}_{g}$ acts cocompactly. This would not be enough, however - one would also have to prove that the simplex stabilizer subgroups were finitely presentable. In other words, this complex would provide the inductive step in a proof that $\mathcal{I}_{g}$ was finitely presentable, but one would still need a base case.

A complex that does not work We close this introduction by discussing an approach to Theorem B that does not work. One might think of trying to prove Theorem B using the following complex. Let $a \in \mathrm{H}_{1}\left(\Sigma_{g} ; \mathbb{Z}\right)$ be a primitive vector. Define $\mathcal{C}_{a}$ to be the graph whose vertices are isotopy classes of oriented simple closed curves $\gamma$ on $\Sigma_{g}$ such that $[\gamma]=a$ and where two vertices $\gamma$ and $\gamma^{\prime}$ are joined by an edge if 
$i_{g}\left(\gamma, \gamma^{\prime}\right)=0$. It is known ([17, Theorem 1.9]; see [12] for an alternate proof) that $\mathcal{C}_{a}$ is connected for $g \geq 3$. Moreover, $\mathcal{I}_{g}$ acts transitively on the vertices of $\mathcal{C}_{a}$. However, it does not act cocompactly; indeed, there are infinitely many edge orbits. To see this, consider edges $e_{1}=\left\{\gamma_{1}, \gamma_{1}^{\prime}\right\}$ and $e_{2}=\left\{\gamma_{2}, \gamma_{2}^{\prime}\right\}$ of $\mathcal{C}_{a}$. Assume that there exists some $f \in \mathcal{I}_{g}$ such that $f\left(e_{1}\right)=e_{2}$. Since $\gamma_{1}$ is homologous to $\gamma_{1}^{\prime}$, the multicurve $\gamma_{1} \cup \gamma_{1}^{\prime}$ divides $\Sigma_{g}$ into two subsurfaces $S_{1}$ and $S_{1}^{\prime}$. Similarly, $\gamma_{2} \cup \gamma_{2}^{\prime}$ divides $\Sigma_{g}$ into two subsurfaces $S_{2}$ and $S_{2}^{\prime}$. Relabeling if necessary, we have $f\left(S_{1}\right)$ isotopic to $S_{2}$ and $f\left(S_{1}^{\prime}\right)$ isotopic to $S_{2}^{\prime}$. Since $f \in \mathcal{I}_{g}$, the images of $\mathrm{H}_{1}\left(S_{1} ; \mathbb{Z}\right)$ and $\mathrm{H}_{1}\left(S_{2} ; \mathbb{Z}\right)$ in $\mathrm{H}_{1}\left(\Sigma_{g} ; \mathbb{Z}\right)$ must be the same, and similarly for $\mathrm{H}_{1}\left(S_{1}^{\prime} ; \mathbb{Z}\right)$ and $\mathrm{H}_{1}\left(S_{2}^{\prime} ; \mathbb{Z}\right)$. It is easy to see that infinitely many such images occur for different edges of $\mathcal{C}_{a}$, so there must be infinitely many edges orbits. We remark that Johnson proved in [8, Corollary to Lemma 9 on page 250$]$ that the images of $\mathrm{H}_{1}\left(S_{1} ; \mathbb{Z}\right)$ and $\mathrm{H}_{1}\left(S_{1}^{\prime} ; \mathbb{Z}\right)$ in $\mathrm{H}_{1}\left(\Sigma_{g} ; \mathbb{Z}\right)$ are a complete invariant for the edge orbits.

Acknowledgments I wish to thank Tara Brendle, Benson Farb, and Dan Margalit for their help. I also wish to thank an anonymous referee for a very helpful referee report. The author is supported in part by NSF grant DMS-1005318.

\section{The Torelli group on subsurfaces}

We will need to understand how the Torelli group restricts to subsurfaces. For a general discussion of this, see [16]. In this section, we will extract from [16] results on two kinds of subsurfaces. In Section 2.1, we will show how to analyze subsurfaces like the subsurfaces $R_{i j k}$ from Section 1. In Section 2.2, we will show how to analyze stabilizers of nonseparating simple closed curves (which are supported on the subsurface obtained by taking the complement of a regular neighborhood of the curve).

\subsection{Analyzing the subsurfaces $R_{i j k}$}

We begin by defining groups $\mathcal{I}_{g, n}$ for $n \geq 2$. There is a map $\operatorname{Mod}_{g, n} \rightarrow \operatorname{Mod}_{g}$ induced by gluing discs to the boundary components of $\Sigma_{g, n}$ and extending homeomorphisms by the identity. Define $\mathcal{I}_{g, n}$ to be the kernel of the resulting action of $\operatorname{Mod}_{g, n}$ on $\mathrm{H}_{1}\left(\Sigma_{g} ; \mathbb{Z}\right)$. For the case $n=1$, the map $\mathrm{H}_{1}\left(\Sigma_{g, 1} ; \mathbb{Z}\right) \rightarrow \mathrm{H}_{1}\left(\Sigma_{g} ; \mathbb{Z}\right)$ is an isomorphism, so this agrees with our previous definition of $\mathcal{I}_{g, 1}$.

Remark In [16], the different definitions of the Torelli group on a surface with boundary are parametrized by partitions of the boundary components. The above definition of $\mathcal{I}_{g, n}$ corresponds to the discrete partition $\left\{\left\{\beta_{1}\right\}, \ldots,\left\{\beta_{n}\right\}\right\}$ of the set $\left\{\beta_{1}, \ldots, \beta_{n}\right\}$ of boundary components of $\Sigma_{g, n}$. 
In [16, Theorem 1.2], a version of the Birman exact sequence is proven for the Torelli group. For $\mathcal{I}_{g, n}$ with $g \geq 2$, it takes the form

$$
1 \longrightarrow \pi_{1}\left(U \Sigma_{g, n}\right) \longrightarrow \mathcal{I}_{g, n+1} \longrightarrow \mathcal{I}_{g, n} \longrightarrow 1 \text {. }
$$

Here $U \Sigma_{g, n}$ is the unit tangent bundle of $\Sigma_{g, n}$. The subgroup $\pi_{1}\left(U \Sigma_{g, n}\right)$ of $\mathcal{I}_{g, n+1}$ is often called the "disc-pushing subgroup" - the mapping class associated to $\gamma \in$ $\pi_{1}\left(U \Sigma_{g, n}\right)$ "pushes" a fixed boundary component around $\gamma$ while allowing it to rotate. The following is an immediate consequence of (1) and the fact that $\pi_{1}\left(U \Sigma_{g}\right)$ can be generated by $2 g+1$ elements.

Lemma 2.1 $\mathcal{I}_{g, 1}$ can be generated by $k+2 g+1$ elements if $\mathcal{I}_{g}$ can be generated by $k$ elements.

Now assume that $S \cong \Sigma_{h, n}$ is an embedded subsurface of $\Sigma_{g}$ and that all the boundary components of $S$ are non-nullhomotopic separating curves in $\Sigma_{g}$. For example, $S$ could be one of the surfaces $R_{i j k}$ from Section 1. Letting $\operatorname{Mod}(S)$ be the mapping class group of $S$, the induced $\operatorname{map} \operatorname{Mod}(S) \rightarrow \operatorname{Mod}_{g}$ is an injection. This gives a natural identification of $\operatorname{Mod}(S)$ with $\operatorname{Mod}\left(\Sigma_{g}, S\right)$. The group $\mathcal{I}\left(\Sigma_{g}, S\right)$ is thus naturally a subgroup of $\operatorname{Mod}(S) \cong \operatorname{Mod}_{h, n}$, and in [16, Theorem 1.1] it is proven that $\mathcal{I}\left(\Sigma_{g}, S\right)=\mathcal{I}_{h, n}$. Johnson [9] proved that $\mathcal{I}_{3}$ can be generated by 35 elements. Applying (1) repeatedly, we see that $\mathcal{I}_{3,1}$ can be generated by 42 elements, $\mathcal{I}_{3,2}$ by 49 elements, and $\mathcal{I}_{3,3}$ by 57 elements. Since $R_{i j k} \cong \Sigma_{3, k}$ with $k \leq 3$, we obtain the following.

Lemma 2.2 For all $1 \leq i<j<k \leq g$, the group $\mathcal{I}\left(\Sigma_{g}, R_{i j k}\right)$ can be generated by 57 elements.

Remark It is well-known (see, eg [16, Section 2.1]) that the mapping classes corresponding to the generators of $\pi_{1}\left(U \Sigma_{g, n}\right)$ used to prove Lemmas 2.1 and 2.2 can be chosen to be bounding pair maps and separating twists. Additionally, Johnson's minimal-size generating set for $\mathcal{I}_{3}$ consists entirely of bounding pair maps, so the generating set for $\mathcal{I}\left(\Sigma_{g}, R_{i j k}\right)$ in Lemma 2.2 can be taken to consist of bounding pair maps and separating twists.

\subsection{Stabilizers of nonseparating simple closed curves}

Let $\gamma$ be a nonseparating simple closed curve on $\Sigma_{g}$. Define $\Sigma_{g, \gamma}$ to be the result of cutting $\Sigma_{g}$ along $\gamma$, so $\Sigma_{g, \gamma} \cong \Sigma_{g-1,2}$. Letting $\operatorname{Mod}_{g, \gamma}$ be the mapping class group of $\Sigma_{g, \gamma}$, the natural map $\Sigma_{g, \gamma} \rightarrow \Sigma_{g}$ induces a map $i: \operatorname{Mod}_{g, \gamma} \rightarrow \operatorname{Mod}_{g}$. Define $\mathcal{I}_{g, \gamma}=i^{-1}\left(\mathcal{I}_{g}\right)$. The map $i$ restricts to a surjection $\mathcal{I}_{g, \gamma} \rightarrow\left(\mathcal{I}_{g}\right)_{\gamma}$, where $\left(\mathcal{I}_{g}\right)_{\gamma}$ is the stabilizer subgroup of $\gamma$. 
Remark In the notation of [16], the group $\mathcal{I}_{g, \gamma}$ corresponds to the Torelli group of $\Sigma_{g-1,2}$ with respect to the "indiscrete partition" $\left\{\left\{\beta, \beta^{\prime}\right\}\right\}$ of the boundary components $\beta$ and $\beta^{\prime}$ of $\Sigma_{g, \gamma}$. Also, the kernel of the map $\mathcal{I}_{g, \gamma} \rightarrow\left(\mathcal{I}_{g}\right)_{\gamma}$ is isomorphic to $\mathbb{Z}$ and is generated by $T_{\beta} T_{\beta^{\prime}}^{-1}$, where $T_{\beta}$ and $T_{\beta^{\prime}}$ are the Dehn twists about $\beta$ and $\beta^{\prime}$, respectively.

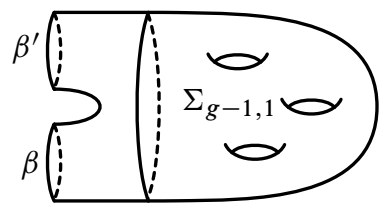

(a)

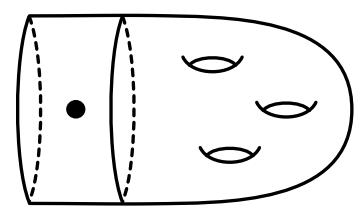

(b)

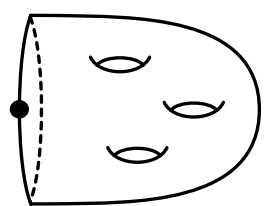

(c)

Figure 2: (a) The surface $\Sigma_{g, \gamma}$ and the subsurface $\Sigma_{g-1,1}$ of $\Sigma_{g, \gamma}$ such that the induced map $\mathcal{I}_{g-1,1} \rightarrow \mathcal{I}_{g, \gamma}$ splits the exact sequence (2) $\quad$ (b) The basepoint for $\pi_{1}\left(\Sigma_{g-1,1}\right)$ is obtained from $\Sigma_{g, \gamma}$ by collapsing the boundary component $\beta$ to a point. (c) The surface in $\mathrm{b}$ deformation retracts to $\Sigma_{g-1,1}$ such that the basepoint ends up on the boundary component.

In [16, Theorem 1.2], it is proven that for $g \geq 2$ there is a short exact sequence

$$
1 \longrightarrow K_{g, \gamma} \longrightarrow \mathcal{I}_{g, \gamma} \longrightarrow \mathcal{I}_{g-1,1} \longrightarrow 1 \text {. }
$$

Here $K_{g, \gamma} \cong\left[\pi_{1}\left(\Sigma_{g-1,1}\right), \pi_{1}\left(\Sigma_{g-1,1}\right)\right]$. This exact sequence splits via the inclusion $\mathcal{I}_{g-1,1} \hookrightarrow \mathcal{I}_{g, \gamma}$ induced by the inclusion $\Sigma_{g-1,1} \hookrightarrow \Sigma_{g, \gamma}$ indicated in Figure 2(a). In other words, the following holds.

Lemma 2.3 $\mathcal{I}_{g, \gamma}=K_{g, \gamma} \ltimes \mathcal{I}_{g-1,1}$ for $g \geq 3$ and $\gamma$ a simple closed nonseparating curve on $\Sigma_{g}$.

The group $\mathcal{I}_{g-1,1}$ acts on $K_{g, \gamma}<\pi_{1}\left(\Sigma_{g-1,1}\right)$ as follows. As is clear from [16, Theorem 1.2], the basepoint for $\pi_{1}\left(\Sigma_{g-1,1}\right)$ is as indicated in Figure 2(b). As shown in Figure 2(c), the surface $\Sigma_{g-1,1}$ deformation retracts onto the surface $\Sigma_{g-1,1}$ on which $\mathcal{I}_{g-1,1}$ is supported. After this deformation retract, the basepoint ends up on $\partial \Sigma_{g-1,1}$. Summing up, $\mathcal{I}_{g-1,1}$ acts on $K_{g, \gamma}<\pi_{1}\left(\Sigma_{g-1,1}\right)$ via the action of $\operatorname{Mod}_{g-1,1}$ on $\pi_{1}\left(\Sigma_{g-1,1}\right)$, where the basepoint for $\pi_{1}\left(\Sigma_{g-1,1}\right)$ is on $\partial \Sigma_{g-1,1}$.

\section{The handle graph is connected}

In this section, we prove the following.

Lemma 3.1 Fix $g \geq 3$. Let $a, b \in \mathrm{H}_{1}\left(\Sigma_{g} ; \mathbb{Z}\right)$ satisfy $i_{a}(a, b)=1$. Then $\mathcal{H}_{a, b}$ is connected. 
We will need two lemmas. In the first, if $\epsilon$ is an oriented arc in a surface, then $\epsilon^{-1}$ denotes the arc obtained by reversing the orientation of $\epsilon$.

Lemma 3.2 Let the boundary components of $\Sigma_{g, 2}$ be $\delta_{0}$ and $\delta_{1}$. Choose points $v_{i} \in \delta_{i}$ for $i=0,1$ and let $\epsilon$ be an oriented properly embedded arc in $\Sigma_{g, 2}$ whose initial point is $v_{0}$ and whose terminal point is $v_{1}$. Then for any $h \in \mathrm{H}_{1}\left(\Sigma_{g, 2} ; \mathbb{Z}\right)$, there exists an oriented properly embedded arc $\epsilon^{\prime}$ in $\Sigma_{g, 2}$ whose initial point is $v_{0}$ and whose terminal point is $v_{1}$ such that the homology class of the loop $\epsilon^{\prime} \cdot \epsilon^{-1}$ is $h$.

Proof Gluing $\left(\delta_{0}, v_{0}\right)$ to $\left(\delta_{1}, v_{1}\right)$, we obtain a surface $S \cong \Sigma_{g+1}$. Let $\alpha$ and $*$ be the images of $\delta_{0}$ and $v_{0}$ in $S$, respectively. The image of $\epsilon$ in $S$ is an oriented simple closed curve $\beta$ with $i_{g}(\alpha, \beta)=1$. There is a natural isomorphism $\mathrm{H}_{1}\left(\Sigma_{g, 2} ; \mathbb{Z}\right) \cong[\alpha]^{\perp}$, where the orthogonal complement is taken with respect to $i_{a}(\cdot, \cdot)$. Under this identification, we can apply [16, Lemma A.3] to find an oriented simple closed curve $\beta^{\prime}$ on $S$ such that $\left[\beta^{\prime}\right]=[\beta]+h$ and such that $\alpha \cap \beta^{\prime}=\{*\}$. Cutting $S$ open along $\alpha$, the curve $\beta^{\prime}$ becomes the desired arc $\epsilon^{\prime}$.

Lemma 3.3 Let $a, b \in \mathrm{H}_{1}\left(\Sigma_{g} ; \mathbb{Z}\right)$ satisfy $i_{a}(a, b)=1$. Let $\alpha_{1}$ and $\alpha_{2}$ be disjoint oriented simple closed curves on $\Sigma_{g}$ such that $\left[\alpha_{i}\right]=a$ for $i=1,2$. There then exists some oriented simple closed curve $\beta$ on $\Sigma_{g}$ such that $[\beta]=b$ and $i_{g}\left(\alpha_{i}, \beta\right)=1$ for $i=1,2$.

Proof Let $\beta^{\prime}$ be any simple closed curve on $\Sigma_{g}$ such that $i\left(\alpha_{i}, \beta^{\prime}\right)=1$ for $i=1,2$. Orient $\beta^{\prime}$ so that its intersections with $\alpha_{1}$ and $\alpha_{2}$ are positive. Let $X_{1}$ and $X_{2}$ be the two subsurfaces of $\Sigma_{g}$ that result from cutting $\Sigma_{g}$ along $\alpha_{1} \cup \alpha_{2}$. For $i=1,2$, the surface $X_{i}$ has 2 boundary components and the intersection of $\beta^{\prime}$ with $X_{i}$ is an oriented properly embedded $\operatorname{arc} \epsilon_{i}$ running between these boundary components. Also, the induced map $\mathrm{H}_{1}\left(X_{i} ; \mathbb{Z}\right) \rightarrow \mathrm{H}_{1}\left(\Sigma_{g} ; \mathbb{Z}\right)$ is an injection, and we will identify $\mathrm{H}_{1}\left(X_{i} ; \mathbb{Z}\right)$ with its image in $\mathrm{H}_{1}\left(\Sigma_{g} ; \mathbb{Z}\right)$. The orthogonal complement to $a$ with respect to the algebraic intersection pairing is spanned by $\mathrm{H}_{1}\left(X_{1} ; \mathbb{Z}\right) \cup \mathrm{H}_{1}\left(X_{2} ; \mathbb{Z}\right)$. Since $i_{a}(a, b)=i_{a}\left(a,\left[\beta^{\prime}\right]\right)$, the homology class $b-\left[\beta^{\prime}\right]$ is orthogonal to $a$. There thus exist $h_{i} \in \mathrm{H}_{1}\left(X_{i} ; \mathbb{Z}\right)$ for $i=1,2$ such that $b=\left[\beta^{\prime}\right]+h_{1}+h_{2}$. Lemma 3.2 says that for $i=1,2$ there exists an oriented properly embedded arc $\epsilon_{i}^{\prime}$ in $X_{i}$ with the same endpoints as $\epsilon_{i}$ such that the homology class of the loop $\epsilon_{i}^{\prime} \cdot \epsilon_{i}^{-1}$ equals $h_{i}$. Letting $\beta$ be the loop $\epsilon_{1}^{\prime} \cdot \epsilon_{2}^{\prime}$, it follows that $[\beta]=\left[\beta^{\prime}\right]+h_{1}+h_{2}=b$, as desired.

Proof of Lemma 3.1 Let $\delta$ and $\delta^{\prime}$ be vertices of $\mathcal{H}_{a, b}$. We will construct a path in $\mathcal{H}_{a, b}$ from $\delta$ to $\delta^{\prime}$. Without loss of generality, $[\delta]=\left[\delta^{\prime}\right]=a$. By [17, Theorem 1.9] (see [12] for an alternate proof), we can find a sequence

$$
\delta=\alpha_{1}, \alpha_{2}, \ldots, \alpha_{n}=\delta^{\prime}
$$


of isotopy classes of oriented simple closed curves on $\Sigma_{g}$ such that $\left[\alpha_{i}\right]=a$ for $1 \leq i \leq n$ and $i_{g}\left(\alpha_{i}, \alpha_{i+1}\right)=0$ for $1 \leq i<n$ (this is where we use the condition $g \geq 3$ ). Lemma 3.3 implies that there exist isotopy classes $\beta_{1}, \ldots, \beta_{n-1}$ of oriented simple closed curves on $\Sigma_{g}$ such that $\left[\beta_{i}\right]=b$ and $i_{g}\left(\alpha_{i}, \beta_{i}\right)=i_{g}\left(\alpha_{i+1}, \beta_{i}\right)=1$ for $1 \leq i<n$. Since $\beta_{i}$ is adjacent to both $\alpha_{i}$ and $\alpha_{i+1}$ in $\mathcal{H}_{a, b}$, the desired path from $\delta$ to $\delta^{\prime}$ is thus

$$
\delta=\alpha_{1}, \beta_{1}, \alpha_{2}, \beta_{2}, \ldots, \beta_{n-1}, \alpha_{n}=\delta^{\prime} .
$$

\section{Generating the stabilizer of a nonseparating simple closed curve}

Let the subsurfaces $R_{i}^{\prime}$ of $\Sigma_{g}$ be as in the introduction. Define $S_{i}=\overline{\Sigma_{g} \backslash R_{i}^{\prime}}$. The goal of this section is to prove the following lemma.

Lemma 4.1 Assume that $g \geq 4$. Let $\gamma$ be the isotopy class of a simple closed nonseparating curve on $\Sigma_{g}$ that is contained in $R_{1}^{\prime}$. Then the subgroup $\left(\mathcal{I}_{g}\right)_{\gamma}$ of $\mathcal{I}_{g}$ stabilizing $\gamma$ is contained in the subgroup of $\mathcal{I}_{g}$ generated by $\cup_{i=1}^{g} \mathcal{I}\left(\Sigma_{g}, S_{i}\right)$.

Before proving this, we need a technical lemma. Set $\pi=\pi_{1}\left(\Sigma_{g, 1}, *\right)$, where $* \in \partial \Sigma_{g, 1}$. Let $T_{1}^{\prime}, \ldots, T_{g}^{\prime}$ be disjoint subsurfaces of $\Sigma_{g, 1}$ such that $T_{i}^{\prime} \cong \Sigma_{1,1}$ and $T_{i}^{\prime} \cap \partial \Sigma_{g, 1}=\varnothing$ for $1 \leq i \leq g$ (see Figure 3(a)). Define $T_{i}=\overline{\Sigma_{g, 1} \backslash T_{i}^{\prime}}$. We have $T_{i} \cong \Sigma_{g-1,2}$ and $* \in T_{i}$ for $1 \leq i \leq g$. The maps $\pi_{1}\left(T_{i}, *\right) \rightarrow \pi_{1}\left(\Sigma_{g, 1}, *\right)$ and $\mathrm{H}_{1}\left(T_{i}^{\prime} ; \mathbb{Z}\right) \rightarrow$ $\mathrm{H}_{1}\left(\Sigma_{g, 1} ; \mathbb{Z}\right)$ are injective; we will identify $\pi_{1}\left(T_{i}, *\right)$ and $\mathrm{H}_{1}\left(T_{i}^{\prime} ; \mathbb{Z}\right)$ with their images in $\pi_{1}\left(\Sigma_{g, 1}, *\right)$ and $\mathrm{H}_{1}\left(\Sigma_{g} ; \mathbb{Z}\right)$, respectively. Define $K_{i}=[\pi, \pi] \cap \pi_{1}\left(T_{i}, *\right)$. We then have the following.

Lemma 4.2 For $g \geq 3$, the group $[\pi, \pi]$ is generated by the $\mathcal{I}_{g, 1}$-orbits of the set $\cup_{i=1}^{g} K_{i}$.

The proof of this will have two ingredients. The first is the following theorem of Tomaszewski. As notation, if $G$ is a group and $a, b \in G$, then $[a, b]:=a^{-1} b^{-1} a b$ and $a^{b}:=b^{-1} a b$.

Theorem 4.3 (Tomaszewski, [20]) Let $F_{n}$ be the free group on $\left\{x_{1}, \ldots, x_{n}\right\}$. Then the set

$$
\left\{\left[x_{i}, x_{j}\right]^{x_{i}^{k_{i}} x_{i+1}^{k_{i+1}} \cdots x_{n}^{k_{n}}} \mid 1 \leq i<j \leq n \text { and } k_{m} \in \mathbb{Z} \text { for all } i \leq m \leq n\right\}
$$

is a free basis for $\left[F_{n}, F_{n}\right]$. 


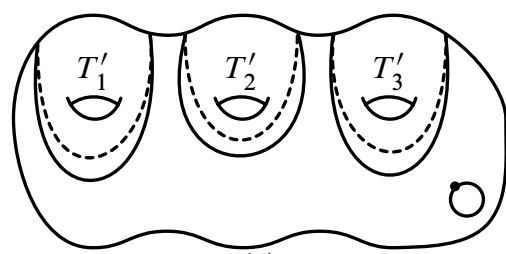

(a)

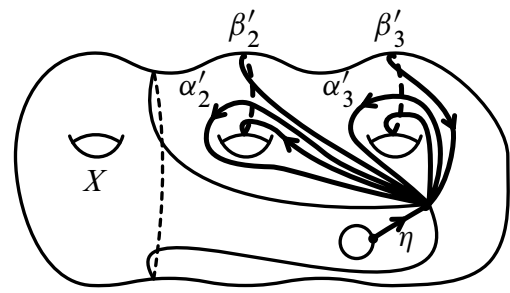

(c)

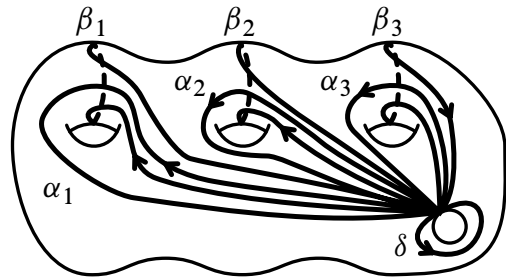

(b)

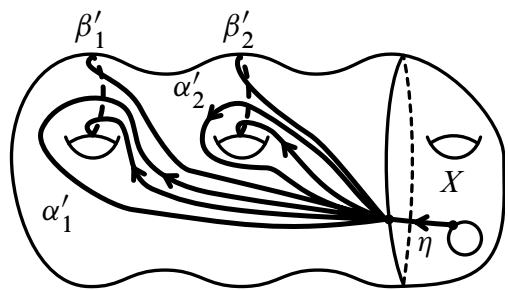

(d)

Figure 3: (a) The subsurfaces $T_{i}^{\prime}$

(b) The standard basis for $\pi$

(c) The surface $X$ when $i=1$

(d) The surface $X$ when $i=g$

The second is the following lemma about the action of $\mathcal{I}_{g, 1}$ on $\pi$. Choose a standard basis $\left\{\alpha_{1}, \beta_{1}, \ldots, \alpha_{g}, \beta_{g}\right\}$ for $\pi$ (as in Figure 3(b)) such that $\alpha_{i}$ and $\beta_{i}$ are freely homotopic into $T_{i}^{\prime}$ for $1 \leq i \leq g$. Our proof of Lemma 4.2 would be much simpler if the image of $\operatorname{Mod}_{g, 1}$ in $\operatorname{Aut}(\pi)$ contained the inner automorphisms - since inner automorphisms act trivially on homology, this would imply that the $\mathcal{I}_{g}$-orbits of $\left\{[x, y] \mid x, y \in\left\{\alpha_{1}, \beta_{1}, \ldots, \alpha_{g}, \beta_{g}\right\}\right\}$ generate $[\pi, \pi]$. However, the image of $\operatorname{Mod}_{g, 1}$ in $\operatorname{Aut}(\pi)$ does not contain the inner automorphisms since $\operatorname{Mod}_{g, 1}$ fixes the loop $\delta=\left[\alpha_{1}, \beta_{1}\right] \cdots\left[\alpha_{g}, \beta_{g}\right]$ depicted in Figure $3(\mathrm{~b})$. The following lemma is a weak replacement for this.

Lemma 4.4 Let $i$ be either 1 or $g$. Consider $h \in \mathrm{H}_{1}\left(T_{i}^{\prime} ; \mathbb{Z}\right)$. There then exists some $w \in\left\langle\alpha_{i}, \beta_{i}, \delta\right\rangle$ and $f \in \mathcal{I}_{g, 1}$ such that $[w]=h$ and such that $f\left(a_{j}\right)=a_{j}^{w}$ and $f\left(b_{j}\right)=b_{j}^{w}$ for $1 \leq j \leq g$ with $j \neq i$.

Proof Let $X$ be a regular neighborhood of the curves $\alpha_{i} \cup \beta_{i} \cup \partial \Sigma_{g, 1}$ depicted in Figure 3(b). Thus $X \cong \Sigma_{1,2}$, the surface $T_{i}^{\prime}$ is homotopic into $X$, and the image of $\pi_{1}(X, *)$ in $\pi$ is $\left\langle\alpha_{i}, \beta_{i}, \delta\right\rangle$. Let $Y=\overline{\Sigma_{g, 1} \backslash X}$, so $Y \cong \Sigma_{g-1,1}$ and $X \cap Y \cong S^{1}$. The key property of $X$ is as follows (this is where we use the assumption that $i$ is either 1 or $g$ ). There exists some $*^{\prime} \in X \cap Y$, a properly embedded arc $\eta$ in $X$ from $*$ to $*^{\prime}$, and elements

$$
\left\{\alpha_{j}^{\prime}, \beta_{j}^{\prime} \mid 1 \leq j \leq g, j \neq i\right\} \subset \pi_{1}\left(Y, *^{\prime}\right)
$$

such that $\alpha_{j}=\eta \cdot \alpha_{j}^{\prime} \cdot \eta^{-1}$ and $\beta_{j}=\eta \cdot \beta_{j}^{\prime} \cdot \eta^{-1}$ for $1 \leq j \leq g$ with $j \neq i$. See Figure 3(c) for the case $i=1$ and Figure 3(d) for the case $i=g$. 
By Lemma 3.2, there exists an oriented properly embedded arc $\eta^{\prime}$ in $X$ whose endpoints are the same as those of $\eta$ such that the homology class of $w:=\eta \cdot\left(\eta^{\prime}\right)^{-1} \in \pi$ in $\mathrm{H}_{1}\left(\Sigma_{g} ; \mathbb{Z}\right)$ is $h$. Observe that $w \in\left\langle\alpha_{i}, \beta_{i}, \delta\right\rangle$. Also,

$$
\eta^{\prime} \cdot \alpha_{j}^{\prime} \cdot\left(\eta^{\prime}\right)^{-1}=w^{-1} \cdot \eta \cdot \alpha_{j}^{\prime} \cdot \eta^{-1} \cdot w=\alpha_{j}^{w}
$$

for $j \neq i$, and similarly for $\beta_{j}$. It is thus enough find some $f \in \mathcal{I}\left(\Sigma_{g}, X\right)$ such that $f(\eta)=\eta^{\prime}$.

The "change of coordinates principle" from [5, Section 1.3] implies that there exists some $f^{\prime} \in \operatorname{Mod}\left(\Sigma_{g}, X\right)$ such that $f^{\prime}(\eta)=\eta^{\prime}$. Briefly, an Euler characteristic calculation shows that cutting $X$ open along either $\eta$ or $\eta^{\prime}$ results in a surface homeomorphic to $\Sigma_{1,1}$. Choosing an orientation-preserving homeomorphism between these two cutopen surfaces and gluing the boundary components back together in an appropriate way, we obtain some $f^{\prime} \in \operatorname{Mod}\left(\Sigma_{g}, X\right)$ such that $f^{\prime}(\eta)=\eta^{\prime}$. See [5, Section 1.3] for more details and many other examples of arguments of this form.

The mapping class $f^{\prime}$ need not lie in Torelli; however, it satisfies $f^{\prime}\left(\left[\alpha_{j}\right]\right)=\left[\alpha_{j}\right]$ and $f^{\prime}\left(\left[\beta_{j}\right]\right)=\left[\beta_{j}\right]$ for $j \neq i$ and $f^{\prime}\left(\mathrm{H}_{1}\left(T_{i}^{\prime} ; \mathbb{Z}\right)\right)=\mathrm{H}_{1}\left(T_{i}^{\prime} ; \mathbb{Z}\right)$. Since the image of $\operatorname{Mod}\left(T_{i}^{\prime}\right)$ in $\operatorname{Aut}\left(\mathrm{H}_{1}\left(T_{i}^{\prime} ; \mathbb{Z}\right)\right)=\operatorname{Aut}\left(\mathbb{Z}^{2}\right)$ is $\mathrm{SL}_{2}(\mathbb{Z})$, we can choose some $f^{\prime \prime} \in$ $\operatorname{Mod}\left(\Sigma_{g}, T_{i}^{\prime}\right)$ such that $f^{\prime}\left(\left[\alpha_{i}\right]\right)=f^{\prime \prime}\left(\left[\alpha_{i}\right]\right)$ and $f^{\prime}\left(\left[\beta_{i}\right]\right)=f^{\prime \prime}\left(\left[\beta_{i}\right]\right)$. It follows that $f:=f^{\prime} \cdot\left(f^{\prime \prime}\right)^{-1}$ lies in $\mathcal{I}\left(\Sigma_{g}, X\right)$ and satisfies $f(\eta)=\eta^{\prime}$, as desired.

Proof of Lemma 4.2 The generating set for $\left[F_{n}, F_{n}\right]$ in Theorem 4.3 depends on an ordering of the generators for $F_{n}$. It seems hard to prove the lemma using the generating set corresponding to the standard ordering

$$
\left(x_{1}, x_{2}, \ldots, x_{2 g}\right)=\left(\alpha_{1}, \beta_{1}, \ldots, \alpha_{g}, \beta_{g}\right)
$$

of the generators for $\pi \cong F_{2 g}$. However, consider the following nonstandard ordering on the generators for $\pi$ :

$$
\left(x_{1}, x_{2}, \ldots, x_{2 g}\right)=\left(\alpha_{2}, \beta_{2}, \alpha_{1}, \beta_{1}, \alpha_{3}, \beta_{3}, \alpha_{4}, \beta_{4}, \ldots, \alpha_{g}, \beta_{g}\right) .
$$

Let $S$ be the generating set for $[\pi, \pi]$ given by Theorem 4.3 using this ordering of the generators. All the elements of $S$ lie in $K_{2}$ except for

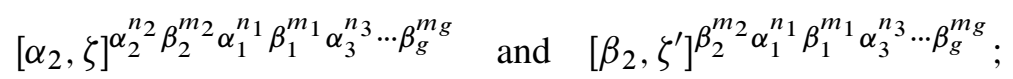

here $\zeta \in\left\{\beta_{2}, \alpha_{1}, \beta_{1}, \alpha_{3}, \ldots, \beta_{g}\right\}$ and $\zeta^{\prime} \in\left\{\alpha_{1}, \beta_{1}, \alpha_{3}, \ldots, \beta_{g}\right\}$ and $n_{i}, m_{i} \in \mathbb{Z}$. Letting $T \subset S$ be the elements in (3), we must show that every $t \in T$ can be expressed as a product of elements in the $\mathcal{I}_{g, 1}$-orbit of the set $\cup_{i=1}^{g} K_{i}$. Consider $t \in T$, so either

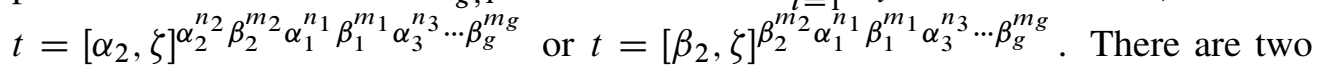
cases. 
Case $1 \zeta \notin\left\{\alpha_{1}, \beta_{1}\right\}$.

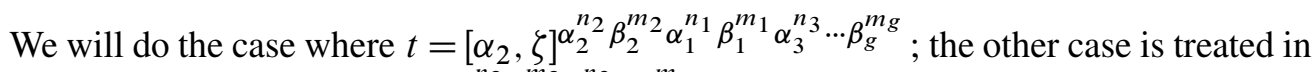
a similar way. Set $t^{\prime}=\left[\alpha_{2}, \zeta\right]^{\alpha_{2}^{n_{2}} \beta_{2}^{m_{2}} \alpha_{3}^{n_{3}} \ldots \beta_{g}^{m g}}$, so $t^{\prime} \in K_{1}$. By Lemma 4.4, there exists some $w \in\left\{\alpha_{1}, \beta_{1}, \delta\right\}$ and $f \in \mathcal{I}_{g, 1}$ such that $[w]=\left[\alpha_{1}^{n_{1}} \beta_{1}^{m_{1}}\right]$ and such that $f\left(a_{j}\right)=a_{j}^{w}$ and $f\left(b_{j}\right)=b_{j}^{w}$ for $j>1$. This implies that $f\left(t^{\prime}\right)=\left[\alpha_{2}, \zeta\right]^{\alpha_{2}^{n_{2}} \beta_{2}^{m_{2}} \alpha_{3}^{n_{3}} \cdots \beta_{g}^{m g} w}$. Now, $\alpha_{3}^{n_{3}} \cdots \beta_{g}^{m_{g}} w$ and $\alpha_{1}^{n_{1}} \beta_{1}^{m_{1}} \alpha_{3}^{n_{3}} \cdots \beta_{g}^{m_{g}}$ are homologous, so there exists some $\theta \in[\pi, \pi]$ such that $\alpha_{3}^{n_{3}} \cdots \beta_{g}^{m_{g}} w \theta=\alpha_{1}^{n_{1}} \beta_{1}^{m_{1}} \alpha_{3}^{n_{3}} \cdots \beta_{g}^{m_{g}}$. Moreover, since $w \in\left\langle a_{1}, b_{1}, \delta\right\rangle$ we have $\theta \in K_{2}$. Observe now that

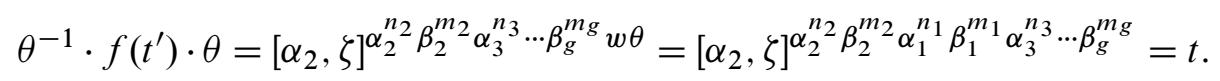

We have thus found the desired expression for $t$.

Case $2 \zeta^{\prime} \in\left\{\alpha_{1}, \beta_{1}\right\}$.

This case is similar to Case 1 . The only difference is that the $\alpha_{g}^{n_{g}} \beta_{g}^{m_{g}}$ term of $t$ is deleted to form $t^{\prime}$ instead of the $\alpha_{1}^{n_{1}} \beta_{1}^{m_{1}}$ term.

Proof of Lemma 4.1 Let $I$ be the subgroup of $\mathcal{I}_{g}$ generated by $\cup_{i=1}^{g} \mathcal{I}\left(\Sigma_{g}, S_{i}\right)$. Using the notation of Section 2, there is a surjection $\rho: \mathcal{I}_{g, \gamma} \rightarrow\left(\mathcal{I}_{g}\right)_{\gamma}$ induced by a continuous map $\phi: \Sigma_{g, \gamma} \rightarrow \Sigma_{g}$. Define $X=\phi^{-1}\left(S_{1}\right)$, so $X \cong \Sigma_{g-1,1}$. Letting $\mathcal{I}(X)$ be the Torelli group of $X$, Lemma 2.3 gives a decomposition $\mathcal{I}_{g, \gamma}=K_{g, \gamma} \ltimes \mathcal{I}(X)$. Clearly $\rho(\mathcal{I}(X))=\mathcal{I}\left(\Sigma_{g}, S_{1}\right) \subset I$. Also, Lemma 4.2 implies that $K_{g, \gamma}$ is generated by the $\mathcal{I}(X)$-conjugates of a set $S \subset K_{g, \gamma}$ such that $\rho(S) \subset I$. We conclude that $\rho\left(\mathcal{I}_{g, \gamma}\right) \subset I$, as desired.

\section{Proof of main theorem}

We finally prove our main theorem. The key is the following standard lemma, whose proof is similar to that given in [19, (1) of Appendix to Section 3] and is thus omitted.

Lemma 5.1 Consider a group $G$ acting without inversions on a connected graph $X$. Assume that $X / G$ consists of a single edge $\bar{e}$. Let $e$ be a lift of $\bar{e}$ to $X$ and let $v$ and $v^{\prime}$ be the endpoints of $e$. Then $G$ is generated by $G_{v} \cup G_{v^{\prime}}$.

To apply this, we will need the following lemma.

Lemma 5.2 Let $a, b \in \mathrm{H}_{1}\left(\Sigma_{g} ; \mathbb{Z}\right)$ satisfy $i_{a}(a, b)=1$. Then $\mathcal{H}_{a, b} / \mathcal{I}_{g}$ is isomorphic to a graph with a single edge. 
The proof is similar to the proofs of [16, Lemma 6.2] and [18, Lemma 6.9], and is thus omitted.

Proof of Theorem B Let $R_{1}^{\prime}, \ldots, R_{g}^{\prime}$ and $R_{i j k}$ be the subsurfaces of $\Sigma_{g}$ from the introduction. Let $\Gamma$ be the subgroup of $\mathcal{I}_{g}$ generated by $\bigcup_{1 \leq i<j<k \leq g} \mathcal{I}\left(\Sigma_{g}, R_{i j k}\right)$. Our goal is to prove that $\Gamma=\mathcal{I}_{g}$.

The proof will be by induction on $g$. The base case $g=3$ is trivial, so assume that $g \geq 4$ and that the theorem is true for all smaller $g$ such that $g \geq 3$. Choose simple closed curves $\alpha$ and $\beta$ in $R_{1}^{\prime}$ such that $i_{g}(\alpha, \beta)=1$. Observe that $R_{1}^{\prime}$ is a closed regular neighborhood of $\alpha \cup \beta$. Set $a=[\alpha]$ and $b=[\beta]$. Clearly $\mathcal{I}_{g}$ acts on $\mathcal{H}_{a, b}$ without inversions. Lemmas 3.1 and 5.2 show that the action of $\mathcal{I}_{g}$ on $\mathcal{H}_{a, b}$ satisfies the other conditions of Lemma 5.1. We deduce that $\mathcal{I}_{g}$ is generated by the union $\left(\mathcal{I}_{g}\right)_{\alpha} \cup\left(\mathcal{I}_{g}\right)_{\beta}$ of the stabilizer subgroups of $\alpha$ and $\beta$.

Recall that $S_{i}=\overline{\Sigma_{g} \backslash R_{i}^{\prime}}$ for $1 \leq i \leq g$. By Lemma 4.1, both $\left(\mathcal{I}_{g}\right)_{\alpha}$ and $\left(\mathcal{I}_{g}\right)_{\beta}$ are contained in the subgroup generated by $\cup_{i=1}^{g} \mathcal{I}\left(\Sigma_{g}, S_{i}\right)$. We must prove that $\mathcal{I}\left(\Sigma_{g}, S_{i}\right) \subset \Gamma$ for $1 \leq i \leq g$. We will do the case $i=g$; the other cases are similar. We have a Birman exact sequence

$$
1 \longrightarrow \pi_{1}\left(U \Sigma_{g-1}\right) \longrightarrow \mathcal{I}\left(\Sigma_{g}, S_{g}\right) \longrightarrow \mathcal{I}_{g-1} \longrightarrow 1 .
$$

By induction, the subset $\bigcup_{1 \leq i<j<k \leq g-1} \mathcal{I}\left(\Sigma_{g}, R_{i j k}\right)$ of $\mathcal{I}\left(\Sigma_{g}, S_{g}\right)$ projects to a generating set for $\mathcal{I}_{g-1}$. Also, it is clear that the disc-pushing subgroup $\pi_{1}\left(U \Sigma_{g-1}\right)$ of $\mathcal{I}\left(\Sigma_{g}, S_{g}\right)$ is generated by elements that lie in $\bigcup_{1 \leq i<j<g} \mathcal{I}\left(\Sigma_{g}, R_{i j g}\right)$. We conclude that $\mathcal{I}\left(\Sigma_{g}, S_{g}\right) \subset \Gamma$, as desired.

\section{References}

[1] J S Birman, On Siegel's modular group, Math. Ann. 191 (1971) 59-68 MR0280606

[2] T Brendle, B Farb, personal communication

[3] K S Brown, Presentations for groups acting on simply-connected complexes, J. Pure Appl. Algebra 32 (1984) 1-10 MR739633

[4] B Farb, Some problems on mapping class groups and moduli space, from: "Problems on mapping class groups and related topics", Proc. Sympos. Pure Math. 74, Amer. Math. Soc., Providence, RI (2006) 11-55 MR2264130

[5] B Farb, D Margalit, A Primer on Mapping Class Groups, to be published by Princeton University Press

[6] R Hain, Fundamental groups of branched coverings and the Torelli group in genus 3, in preparation 
[7] A Hatcher, W Thurston, A presentation for the mapping class group of a closed orientable surface, Topology 19 (1980) 221-237 MR579573

[8] D Johnson, Conjugacy relations in subgroups of the mapping class group and a group-theoretic description of the Rochlin invariant, Math. Ann. 249 (1980) 243-263 MR579104

[9] D Johnson, The structure of the Torelli group. I. A finite set of generators for $\mathcal{I}$, Ann. of Math. (2) 118 (1983) 423-442 MR727699

[10] D Johnson, A survey of the Torelli group, from: "Low-dimensional topology (San Francisco, Calif., 1981)", Contemp. Math. 20, Amer. Math. Soc., Providence, RI (1983) 165-179 MR718141

[11] D Johnson, The structure of the Torelli group. III. The abelianization of $\mathcal{T}$, Topology 24 (1985) 127-144 MR793179

[12] D Margalit, A Hatcher, Generating the Torelli group, in preparation

[13] D McCullough, A Miller, The genus 2 Torelli group is not finitely generated, Topology Appl. 22 (1986) 43-49 MR831180

[14] G Mess, The Torelli groups for genus 2 and 3 surfaces, Topology 31 (1992) 775-790 MR1191379

[15] J Powell, Two theorems on the mapping class group of a surface, Proc. Amer. Math. Soc. 68 (1978) 347-350 MR0494115

[16] A Putman, Cutting and pasting in the Torelli group, Geom. Topol. 11 (2007) 829-865 MR2302503

[17] A Putman, A note on the connectivity of certain complexes associated to surfaces, Enseign. Math. (2) 54 (2008) 287-301 MR2478089

[18] A Putman, An infinite presentation of the Torelli group, Geom. Funct. Anal. 19 (2009) 591-643 MR2545251

[19] J-P Serre, Trees, Springer, Berlin (1980) MR607504 Translated from the French by John Stillwell

[20] W Tomaszewski, A basis of Bachmuth type in the commutator subgroup of a free group, Canad. Math. Bull. 46 (2003) 299-303 MR1981684

Department of Mathematics, Rice University, MS 136, 6100 Main St

Houston, TX 77005, USA

andyp@rice.edu

http://www.math.rice.edu/ andyp/

Proposed: Joan Birman

Received: 24 June 2011

Seconded: Danny Calegari, Ronald Stern

Revised: 11 August 2011 